\title{
Anharmonicity in Raman-active phonon modes in atomically thin $\mathrm{MoS}_{2}$
}

\author{
Suman Sarkar, Indrajit Maity $\odot$, and H. L. Pradeepa $\odot$ \\ Department of Physics, Indian Institute of Science, Bangalore-560012, India \\ Goutham Nayak, Laetitia Marty, Julien Renard, Johann Coraux ๑, Nedjma Bendiab, and Vincent Bouchiat \\ Univ. Grenoble Alpes, CNRS, Grenoble INP, Institut Néel, 38000 Grenoble, France \\ Sarthak Das $\odot$ and Kausik Majumdar \\ Department of Electrical Communication Engineering, Indian Institute of Science, Bangalore 560012, India \\ Manish Jain and Aveek Bid $\odot^{*}$ \\ Department of Physics, Indian Institute of Science, Bangalore 560012, India
}

(Received 2 January 2020; revised manuscript received 28 April 2020; accepted 30 April 2020;

published 12 May 2020)

\begin{abstract}
Phonon-phonon anharmonic effects have a strong influence on the phonon spectrum; most prominent manifestation of these effects are the softening (shift in frequency) and broadening (change in FWHM) of the phonon modes at finite temperature. Using Raman spectroscopy, we studied the temperature dependence of the FWHM and Raman shift of $E_{2 g}^{1}$ and $A_{1 g}$ modes for single-layer and natural bilayer $\mathrm{MoS}_{2}$ over a broad range of temperatures $(8<\mathrm{T}<300 \mathrm{~K})$. Both the Raman shift and FWHM of these modes show linear temperature dependence for $T>100 \mathrm{~K}$, whereas they become independent of temperature for $T<100 \mathrm{~K}$. Using first-principles calculations, we show that three-phonon anharmonic effects intrinsic to the material can account for the observed temperature dependence of the linewidth of both the modes. It also plays an important role in determining the temperature dependence of the frequency of the Raman modes. The observed evolution of the linewidth of the $A_{1 g}$ mode suggests that electron-phonon processes are additionally involved. From the analysis of the temperature-dependent Raman spectra of $\mathrm{MoS}_{2}$ on two different substrates- $\mathrm{SiO}_{2}$ and hexagonal boron nitride - we disentangle the contributions of external stress and internal impurities to these phonon-related processes. We find that the renormalization of the phonon mode frequencies on different substrates is governed by strain and intrinsic doping. Our work establishes the role of intrinsic phonon anharmonic effects in deciding the Raman shift in $\mathrm{MoS}_{2}$ irrespective of substrate and layer number.
\end{abstract}

DOI: 10.1103/PhysRevB.101.205302

\section{INTRODUCTION}

$\mathrm{MoS}_{2}$ is a well-studied two-dimensional transition metal dichalcogenide having a direct band gap in its single layer form [1]. Its discovery [1,2] has opened up new possibilities for the semiconductor industry in terms of next-generation optoelectronics and valleytronics devices. The predicted and observed exotic properties of atomically-thin flakes of $\mathrm{MoS}_{2}-$ direct band gap in the monolayer limit, strong correlations giving rise to three-body trion state observable even at room temperature [3], ambipolar transport [4,5], superconductivity [6,7], highly efficient light-matter interactions [8], and strong valley-selectivity $[9,10]$ - make it a very interesting material from an academic perspective. Optical measurements like Raman and photoluminescence (PL) spectroscopy have become the techniques of choice to probe these properties of $\mathrm{MoS}_{2}$ and other related transition metal dichalcogenides.

These optical properties of these materials are influenced by the presence of intrinsic defects (primarily chalcogenide

\footnotetext{
*aveek@iisc.ac.in
}

vacancies) [11-13], effect of ambient, the substrate, or capping layer $[12,14]$, and temperature $[15,16]$. Analyzing the optical properties thus provides a wealth of information about defect dynamics, various energy transfer processes, Coulomb interactions, influence of electric, magnetic field, and strain on spin and valley splitting of the electronic energy bands [17], and temperature-dependent multiphonon scattering processes [18]. Additionally, valuable information can be gleaned from the peak positions and full width at half maximum (FWHM) of the Raman-active modes about the dielectric environment $[19,20]$, strain effects [21-23], anharmonicity in the lattice potential energy [24-26], thermal expansion [26-28], and thermal conductivity [26,29-31] of these materials. Characterizing the effect of the latter (temperature) is essential to understand the limitations to charge carrier mobility and thermal conductivity, hence the performance of devices based on these materials. To this respect, interactions involving two or more phonons [18] and electron-phonon interactions are essential. Their characteristic temperature dependence allows us to unravel their role.

Even after intense research for more than a decade, an in-depth, combined experimental and theoretical study of 
the temperature evolution of the phonon modes in singleand few-layer $\mathrm{MoS}_{2}$ is missing. Previous studies employed semiquantitative models for the calculation of the temperature dependent Raman shift [32,33]. Two contradictory conclusions have been reported by fitting the experimental data to such models for $300 \mathrm{~K}<T<500 \mathrm{~K}$. Najmei et al. concluded that the three-phonon process is irrelevant in explaining the observed phonon-frequency shifts of Raman active modes, whereas Yang et al. found the three-phonon processes to be more dominant [32,33]. Lanzillo et al. [34] performed firstprinciples molecular dynamics simulations including both the three- and four-phonon processes, predicting a characteristic temperature dependence of the Raman shift. However testing this scenario against experimental data was made difficult by the rather limited resolution $\left(\sim 2 \mathrm{~cm}^{-1}\right)$ of the measured Raman spectra. Studies conducted at high temperatures $(300 \mathrm{~K}<T<450 \mathrm{~K})$ show that the positions of the Raman peaks of single-layer $\mathrm{MoS}_{2}$ are linearly redshifted with increasing temperature [26,34-36]. This shift was attributed primarily to four-phonon processes for the $E_{2 g}^{1}$ mode while for the $A_{1 g}$ mode, thermal expansion was also found to play a significant role [32].

In this paper, we report the results of combined experimental and theoretical studies of the temperature dependence of the Raman shifts and full width at half maximum (FWHM) of Raman-active modes in single-layer (SL) and natural bilayer (NBL) $\mathrm{MoS}_{2}$ samples. We find that at low temperatures, the FWHM and Raman shift are practically independent of $T$ while above $125 \mathrm{~K}$ they vary almost linearly with temperature. Our theoretical calculations, based on density functional perturbation theory [37] (DFPT), faithfully reproduce the observed temperature dependencies of FWHM of the Raman active vibrational modes of SL $\mathrm{MoS}_{2}$ over the entire temperature range. We find that three-phonon anharmonic effects are the predominant factor determining the observed temperature dependence of the FWHM for the two prominent Raman modes in $\mathrm{MoS}_{2}$. We also find that higher order phonon processes contribute significantly to the observed high-temperature softening of phonon modes with the temperature. By comparing the temperature dependence of the FWHM and the Raman shift samples prepared on two types of substrate- $-\mathrm{SiO}_{2} / \mathrm{Si}^{++}$and single crystal hexagonal boron nitride $(\mathrm{hBN})$ - we find that strain and electronic doping only contribute a temperature-independent change of the Raman shifts and FWHMs.

\section{METHODS AND MEASUREMENTS}

$\mathrm{MoS}_{2}$ flakes were exfoliated from single crystals of naturally occurring $\mathrm{MoS}_{2}$ (SPI supplies) on polydimethylsiloxane (PDMS). These were transferred on $290 \mathrm{~nm} \mathrm{SiO} 2 / \mathrm{Si}^{++}$ substrates using the well-established dry-transfer technique $[38,39]$. We also prepared $\mathrm{hBN} / \mathrm{MoS}_{2}$ heterostructures on $\mathrm{SiO}_{2} / \mathrm{Si}^{++}$substrates by sequentially aligning and transferring the different atomic layers. The transfers were carried out in an inert atmosphere inside a glove box using a custombuilt system based on a Thorlabs 3-Axis motorized lineartranslation stage (MTS-Z8) under an optical microscope. During transfers, the speed of approach and retraction of the microscope stage was kept less than $1 \mu \mathrm{m} \mathrm{s}^{-1}$ to avoid
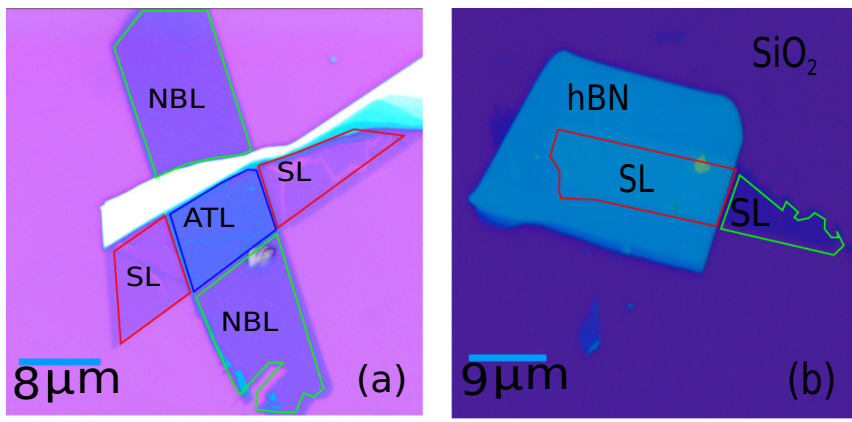

FIG. 1. (a) Optical image of sample1 prepared on $\mathrm{SiO}_{2} / \mathrm{Si}^{++}$ substrate - the SL region is outlined in a solid red line and NBL in a solid green line. (b) Optical image of sample2 prepared on a hBN sample - the $\mathrm{MoS}_{2}$ on $\mathrm{hBN}$ portion is outlined by a solid red line and the $\mathrm{MoS}_{2}$ on $\mathrm{SiO}_{2}$ by a solid green line.

wrinkling and tearing of the flakes. The number of layers in the flakes was initially estimated from the color contrast of the optical images and later confirmed from AFM measurements, Raman spectroscopy, and photoluminescence measurements. We have presented high-frequency and low-frequency Raman measurement along with PL measurement in the Appendix section in Fig. 7 to identify the number of layers in our sample. We also have identified SL and NBL samples with lowfrequency Raman measurement as discussed in the Appendix section in Fig. 8.

We studied two different classes of samples. Multiple samples of each type were studied, and the data on different samples of the same class were qualitatively consistent. In this paper, we focus on the data obtained from a single sample of each class. Figure 1(a) is an optical image of a sample prepared on $\mathrm{SiO}_{2} / \mathrm{Si}^{++}$substrate having SL, NBL, and artificial trilayer (ATL) regions (henceforth referred to as 'sample1'). In this class of samples, we studied the temperature evolution of Raman spectra for the SL and NBL. In the second class of samples, SL MoS 2 was transferred to lie partially on $\mathrm{SiO}_{2}$ and partly on a $\sim 20 \mathrm{~nm}$ thick $\mathrm{hBN}$ flake on the surface of $\mathrm{SiO}_{2}$. An optical image of one such sample (referred to as 'sample2') is shown in Fig. 1(b). These samples were used for the comparative study of the temperature dependence of Raman spectra of SL $\mathrm{MoS}_{2}$ on $\mathrm{SiO}_{2}$ and hBN.

We have performed the low-temperature Raman experiments in reflectance mode on HORIBA Scientific Instrument LABRAM HR Evolution attached with a ARS cryo-free cryostat which can reach $4 \mathrm{~K}$ base temperature. A $532 \mathrm{~nm}$ laser source in our measurement system having a spatial resolution of $1 \mu \mathrm{m}$ has been used. The Raman spectra were recorded using 1800 lines/mm grating at very low laser power levels $(\sim 100 \mu \mathrm{W})$ to avoid heating of the sample. The resolution of our setup is about $1 \mathrm{~cm}^{-1}$. In this report we have concentrated our result over the spectral range $350-450 \mathrm{~cm}^{-1}$ though we have measured the spectra over the range of spectral range 200-800 $\mathrm{cm}^{-1}$. In the Appendix section we have shown in Fig. 6 a typical Raman spectra over the complete range taken at $300 \mathrm{~K}$.

The phonon frequencies, Raman shifts, and FWHM were computed using DFPT [37] and the D3Q code [40] based on density functional theory as implemented in the 

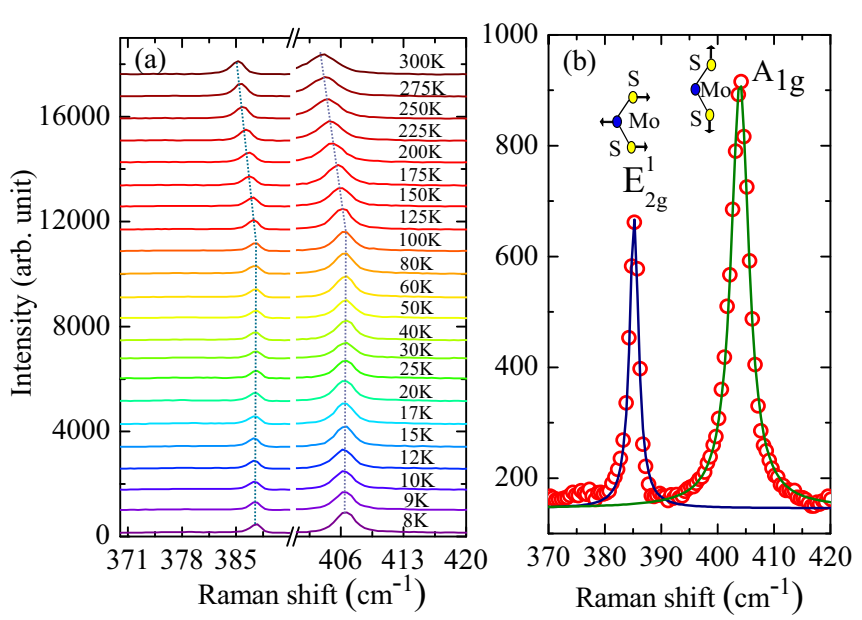

FIG. 2. (a). Raman spectra of sample1 measured at different temperatures between $8 \mathrm{~K}$ and $300 \mathrm{~K}$. The dotted lines are the guide for the eye for marking the evolution of Raman shift with temperature. (b) An individual Raman spectra at $300 \mathrm{~K}$. The blue and olive solid lines are Lorentzian fits to the experimental data. The inset schematics show the $E_{2 g}^{1}$ and $A_{1 g}$ vibrational modes.

QUANTUM ESPRESSO package [41-43]. We used optimized norm-conserving Vanderbilt (ONCV) pseudopotentials $[44,45]$ for Mo and S atoms and the generalized gradient approximation [46] for the exchange correlation. For SL $\mathrm{MoS}_{2}$ and NBL $\mathrm{MoS}_{2}$, we used a plane-waves kinetic energy cutoff of 80 Ry ensuring convergence of phonon frequencies at the $\Gamma$ point within $0.1 \mathrm{~cm}^{-1}$. To avoid interaction between periodic images along the $z$ direction, an $18 \AA$ vacuum spacing was used. For total energy calculations, we used a $12 \times 12 \times 1 \vec{k}$ point sampling of the Brillouin zone, whereas the phonon frequencies, third order force constants, were calculated using a $3 \times 3 \times 1 \vec{k}$ point sampling of the Brillouin zone. Raman shift and FWHM calculations using the third order force constants were performed by summing over discrete uniform $\vec{q}$ grid points $(200 \times 200 \times 1)$ which were randomly shifted from the origin. A Gaussian function with a smearing of $3 \mathrm{~cm}^{-1}$ was used [40] to replace the delta function.

\section{RESULTS AND DISCUSSIONS}

\section{A. Temperature dependence of the lifetimes of Raman modes in $\mathrm{MoS}_{2}$}

High frequency Raman spectrum (we define high frequency in this paper to be the spectral range $350-450 \mathrm{~cm}^{-1}$ ) of SL and NBL $\mathrm{MoS}_{2}$ consists of two Raman active modesdenoted by $E^{\prime}$ and $A_{1}^{\prime}$ for SL and by $E_{2 g}^{1}$ and $A_{1 g}$ for multilayers [47-50]. The $E$ modes arise from in-plane, antiphase oscillations of the two $\mathrm{S}$ atoms with respect to the Mo atom while the $A$ modes are due to the antiphase, out-of-plane oscillations of only the $\mathrm{S}$ atoms [48]. For notational simplicity, we will refer to the in-plane modes as $E_{2 g}^{1}$ and the out-of-plane modes as $A_{1 g}$ for both SL and multilayers. The measured Raman spectra in the range $370-420 \mathrm{~cm}^{-1}$ over the temperature range $8 \mathrm{~K}$ to $300 \mathrm{~K}$ are shown in Fig. 2(a) for the SL region for sample1. The dotted lines are guides to the eye showing the evolution of the peak positions with temperature $T$. We found
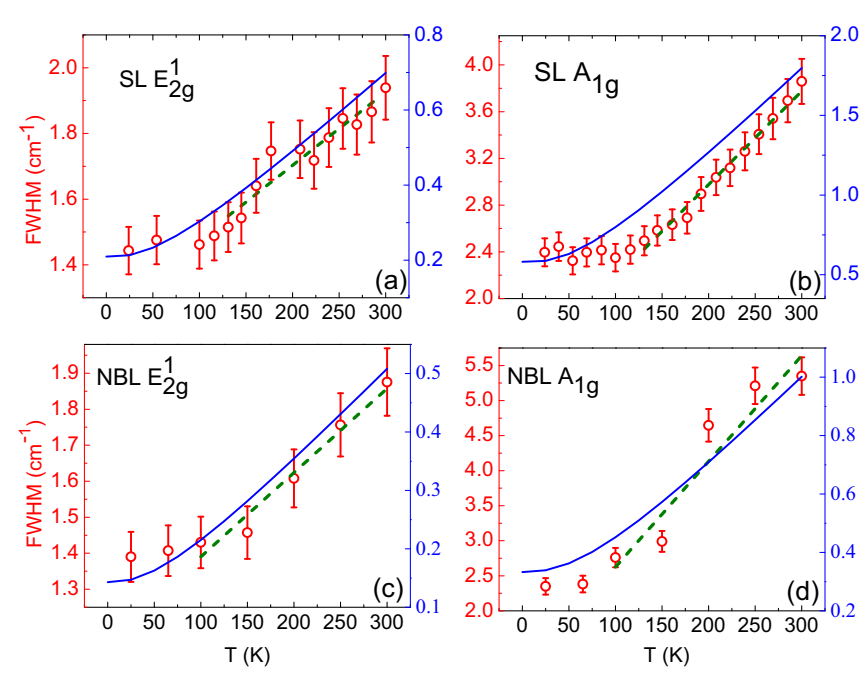

FIG. 3. Temperature dependence of the FWHM of the Raman modes. The experimentally obtained data are plotted on the left axis in open red circles for (a) the $E_{2 g}^{1}$ peak of the SL region, (b) the $A_{1 g}$ peak of the SL region. The corresponding theoretical results are plotted on the right axis in solid blue lines. The green dashed lines are linear fits to the experimental data for $T>100 \mathrm{~K}$. (c), (d) are the corresponding plot for the NBL region. The data are for sample1.

that even in the SL limit, our $\mathrm{MoS}_{2}$ samples have FWHM of about $\sim 1.4 \mathrm{~cm}^{-1}$ and $\sim 2.3 \mathrm{~cm}^{-1}$ for $E_{2 g}^{1}$ and $A_{1 g}$, respectively, which confirms the high crystalline quality of the $\mathrm{MoS}_{2}$ flakes [26].

To extract the peak positions and peak FWHM, we fitted the Raman spectra at every temperature with two Lorentzian peaks. An example is shown in Fig. 2(b) for the data obtained at $300 \mathrm{~K}$. We have also used Voigt fitting to determine the peak position and FWHM shown in the Appendix section in Figs. 9 and 10. We found that in the case of Voigt fitting both Lorentzian width and Gaussian width contribute to the Voigt width and as a result we end up with a minimum FWHM for the $E_{2 g}^{1}$ and $A_{1 g}$ peak, about $\sim 1.8 \mathrm{~cm}^{-1}$ and $\sim 2.5 \mathrm{~cm}^{-1}$, respectively, while from the Lorentzian fit to our Raman spectra the minimum FWHM is about $\sim 1.45 \mathrm{~cm}^{-1}$ and $\sim 2.3 \mathrm{~cm}^{-1}$, respectively, shown in Figs. 10(a) and 10(b), respectively. In Figs. 3(a) and 3(b) we show the temperature dependence of the FWHM of the $E_{2 g}^{1}$ and $A_{1 g}$ mode, respectively, for the SL region of sample1. We observe that the FWHM decreases with decreasing $T$ and tends to saturate below $100 \mathrm{~K}$. The same trend is seen for the FWHM of the $E_{2 g}^{1}$ and $A_{1 g}$ modes measured on the NBL portion of sample 1 - the data are plotted in Figs. 3(c) and 3(d), respectively.

Raman modes have a finite FWHM due to both intrinsic phonon scattering processes and extrinsic factors (linked to defects, the finite size of crystals, etc). At a finite temperature, the intrinsic FWHM of the Raman modes $\gamma^{\text {in }}$ are determined by both electron-phonon interactions and phonon-phonon anharmonic effects, i.e., $\gamma^{\text {in }}=\gamma^{\mathrm{e}-\mathrm{ph}}+\gamma^{\mathrm{ph}-\mathrm{ph}}[26,51]$. Extrinsic factors linked to defect dynamics can cause broadening of the Raman lines in several possible ways-by changing the anharmonicity, causing local fluctuations of the frequency of optical phonons, causing phonon confinement-induced relaxation of the Raman wave vector selection rules, or leading 
TABLE I. Temperature coefficient of Raman shift $(\alpha)$ and FWHM $(\beta)$ for SL and NBL on $\mathrm{SiO}_{2}$ for the two Raman modes of MoS 2 .

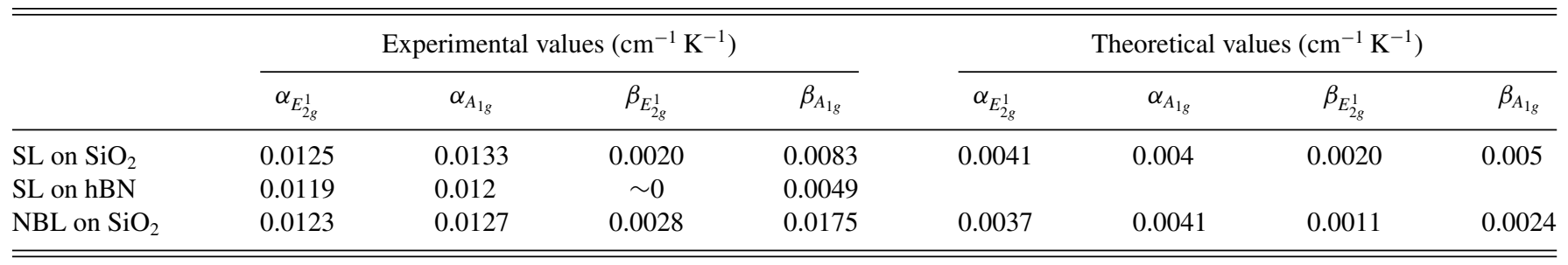

to a change of phonon wave function as the solution of the dynamic problem [52].

We compute the FWHM using third-order phonon-phonon anharmonic effects for both SL and NBL samples (the details of the calculations are given in the following section). The calculated change in FWHM over the temperature range $0<$ $T<300 \mathrm{~K}$ of SL $\mathrm{MoS}_{2}\left(\sim 0.48 \mathrm{~cm}^{-1}\right.$ and $1.2 \mathrm{~cm}^{-1}$ and for $E_{2 g}^{1}$ and $A_{1 g}$, respectively) matches quite well with our measured values $\left(\sim 0.5 \mathrm{~cm}^{-1}\right.$ and $1.55 \mathrm{~cm}^{-1}$ for $E_{2 g}^{1}$ and $A_{1 g}$, respectively). The data are plotted in Figs. 3(a) and 3(b), respectively, for the two modes.

Just like the experimental data the calculated temperature dependence of FWHM of both $A_{1 g}$ and $E_{2 g}^{1}$ modes for SL $\mathrm{MoS}_{2}$ saturates below $100 \mathrm{~K}$ and increases linearly for $T>$ $100 \mathrm{~K}$ with a coefficient we call $\beta$. The calculated and measured values of $\beta$ of the $E_{2 g}^{1}$ and $A_{1 g}$ peaks for SL and NBL are presented in Table I; while the calculated value of $\beta$ is in good agreement with experiment for in-plane vibrational mode, it is underestimated for the out-of-plane mode. This discrepancy cannot be assigned to four-phonon or higher order phonon processes. If four-phonon or higher order phonon processes played an important role then both the modes should have shown the discrepancy. In contrast, the electron-phonon process mostly affects the $A_{1 g}$ modes by reducing their lifetime, hence by increasing the FWHM. Naturally, our calculations addressing the case of undoped $\mathrm{MoS}_{2}$ cannot properly account for the observed electron-phonon interaction-related increase of the FWHM.

\section{B. Temperature dependence of frequency of Raman modes in $\mathrm{MoS}_{2}$}

The experimentally measured temperature dependence of the Raman shifts of the $E_{2 g}^{1}$ and $A_{1 g}$ modes from $300 \mathrm{~K}$ down to $10 \mathrm{~K}$ in the SL region of sample1 are shown in Figs. 4(a) and 4(b), respectively. The corresponding data for the NBL region of sample1 are shown in Figs. 4(c) and 4(d), respectively. We find that above $100 \mathrm{~K}$, the Raman shift of both the $E_{2 g}^{1}$ and $A_{1 g}$ modes, in both SL and NBL, decrease with temperature. Below $100 \mathrm{~K}$, the Raman shift in all cases saturates. Over the temperature range from $100 \mathrm{~K}$ to $300 \mathrm{~K}$ the Raman shifts can be fitted to a relation:

$$
\omega(T)=\omega_{0}-\alpha T
$$

Here $\omega_{0}$ is the peak position extrapolated to zero temperature and $\alpha=d \omega / d T$ is the temperature coefficient of the Raman shift. From linear fits to the data for $T>100 \mathrm{~K}$, we obtain, for both SL and NBL, $\alpha-\sim 0.013 \mathrm{~cm}^{-1} / \mathrm{K}$ for the $E_{2 g}^{1}$ mode and $\sim 0.012 \mathrm{~cm}^{-1} / \mathrm{K}$ for the $A_{1 g}$ mode. In the Appendix section, we have shown that the Voigt fitting to the Raman spectra does not gave any appreciable difference to the peak position shown in Figs. 9(a) and 9(b).

To understand the temperature dependence of the Raman shifts, note that at $T \neq 0 \mathrm{~K}$, phonons frequencies get renormalized due to anharmonic effects. At constant (external) pressure, the corresponding temperature dependence of the phonon frequencies $\omega$ for a semiconductor can be expressed as $\omega(T)-\omega(0)=\Delta \omega_{T}(T)+\Delta \omega_{V}(T)$, where $\Delta \omega_{T}$ and $\Delta \omega_{V}$ are the frequency shifts due to lattice thermal expansion and 'pure' thermal anharmonic effects, which typically include third- and fourth-order phonon-phonon anharmonic effects [53-55]. We compute the frequency shifts by including both the lattice thermal expansion effects [32] and phonon-phonon anharmonic effects, using three-phonon processes. Two kinds of scattering processes are taken into account: (a) a phonon of momentum $\vec{q}$ can decay into two phonons $\left(-\vec{q}^{\prime},-\overrightarrow{q^{\prime \prime}}\right)$, (b) a phonon with $\vec{q}$ can coalesce with a phonon with $-\vec{q}^{\prime}$ to eject one with $-\overrightarrow{q^{\prime \prime}}$. In our first principles based calculations we incorporated all such possible three-phonon processes without any restriction $[40,54]$.

In Figs. 4(a) and 4(b), we plot, on the right axis, the calculated temperature dependence of the $E_{2 g}^{1}$ and $A_{1 g} \mathrm{Ra}$ man shifts for SL $M_{0} S_{2}$. For $T \gtrsim 100 \mathrm{~K}$, the Raman modes soften linearly with $T$ while for $T \lesssim 100 \mathrm{~K}$, the Raman shifts

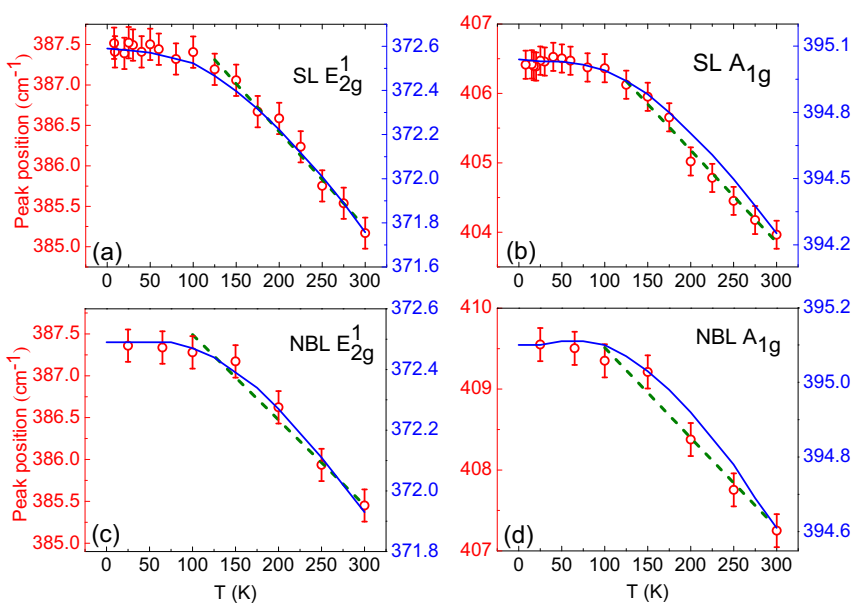

FIG. 4. Temperature dependence of the Raman shift. The experimentally obtained data are plotted on the left axis in open red circles for (a) the $E_{2 g}^{1}$ peak of the SL region, (b) the $A_{1 g}$ peak of the SL region, (c) the $E_{2 g}^{1}$ peak of the NBL region, and (d) the $A_{1 g}$ region of the NBL sample. The corresponding theoretical results are plotted on the right axis in solid blue lines. The measurements were performed on sample1. The green dashed lines are the linear fits to experimental data for $T>100 \mathrm{~K}$. 
saturate and become independent of $T$. This theoretically computed trend captures correctly the experimental temperature dependence.

The temperature dependencies of Raman shifts and FWHM arise from the occupation probabilities of the phonons (Bose factor) [40,54]. In order to get an intuitive understanding of the temperature dependence of the Raman shift, let us consider one possible three-phonon decay channel: an optical phonon with energy $\hbar \omega_{0}$ at $\Gamma$ point decays into two acoustic phonons from the same branch while conserving both energy and momentum [55]. Therefore, the acoustic phonon modes have $\hbar \omega_{0} / 2$ energy with equal and opposite momentum. This process gives rise to a temperature dependent Raman shift of the following form: $\Delta \omega(T) \sim\left[1+\frac{2}{e^{\hbar \omega_{0} / 2 k_{B} T}-1}\right][53,54]$. For both the $E_{2 g}^{1}$ and $A_{1 g}$ modes, $\hbar \omega_{0} \approx 50 \mathrm{meV}$. Thus, at low $T$ $\Delta \omega$ saturates to a constant value and becomes linear in $T$ at higher temperature.

Note however, that the absolute values of the calculated mode frequencies differ from the experimentally measured ones by about $4 \%$. The frequency values are in fact sensitive to the choice of the exchange-correlation functional. Generalized gradient application (GGA) overestimates the lattice constant. For example, with GGA at $0 \mathrm{~K}$ the in-plane lattice constant is $3.18 \AA$, which is $\sim 0.95 \%$ larger than the experimentally measured value [56], and the frequencies of the $E^{\prime}$ and $A_{1}$ bands of SL are $373.7 \mathrm{~cm}^{-1}$ and $396.2 \mathrm{~cm}^{-1}$ while the experimental values at $8 \mathrm{~K}$ are $385.5 \mathrm{~cm}^{-1}$ and $406.5 \mathrm{~cm}^{-1}$.

Linear fits to the calculated $T$ dependence of Raman shifts yield a temperature coefficient $\alpha \sim 0.004 \mathrm{~cm}^{-1} / \mathrm{K}$ for both the $E_{2 g}^{1}$ and $A_{1 g}$ modes (Table I). This value is significantly lower than the experimental values. This underestimation can arise from several parameters (both intrinsic and extrinsic), which are discussed below.

Among the intrinsic phonon-phonon anharmonic effects ignored in our calculations, the principal one is a four-phonon process. It has been proposed that the relative contribution to Raman shifts at finite $T$ from the three-phonon and fourphonon processes is related to the phonon band gap of the material. The larger the band gap, the greater the contribution from four-phonon processes [57]. The band gap between acoustic and optical modes in SL $\mathrm{MoS}_{2}$ is $\sim 6 \mathrm{meV}$, which is rather low. Thus, we expect that the phase space available for three-phonon decay channels is non-negligible compared to higher order four-phonon processes. A significant contribution to the Raman shift temperature dependence from fourphonon and higher-phonon processes could thus be expected. Although extrinsic effects linked to defects [58] or the substrate [59], adsorbates, and fabrication induced disorder [60] in a sample can give a constant shift to the Raman shift and FWHM of the Raman modes, we show in the next section that contribution to their temperature dependence is marginal.

Compared to single layer, natural bilayer shows a redshift in the $E_{2 g}^{1}$ Raman shift and a blueshift in the $A_{1 g}$ Raman shift [50]. The blueshift in the $A_{1 g}$ mode can account for an additional springlike interaction, related to short-ranged interaction involving the nearest neighbor $\mathrm{S}$ atom. On the other hand, the redshift in the $E_{2 g}^{1}$ can be attributed to a greater dielectric screening of the Coulomb forces in NBL $\mathrm{MoS}_{2}$ [47] compared to the case of single layer. The trends of the calculated temperature dependence of Raman shifts for NBL
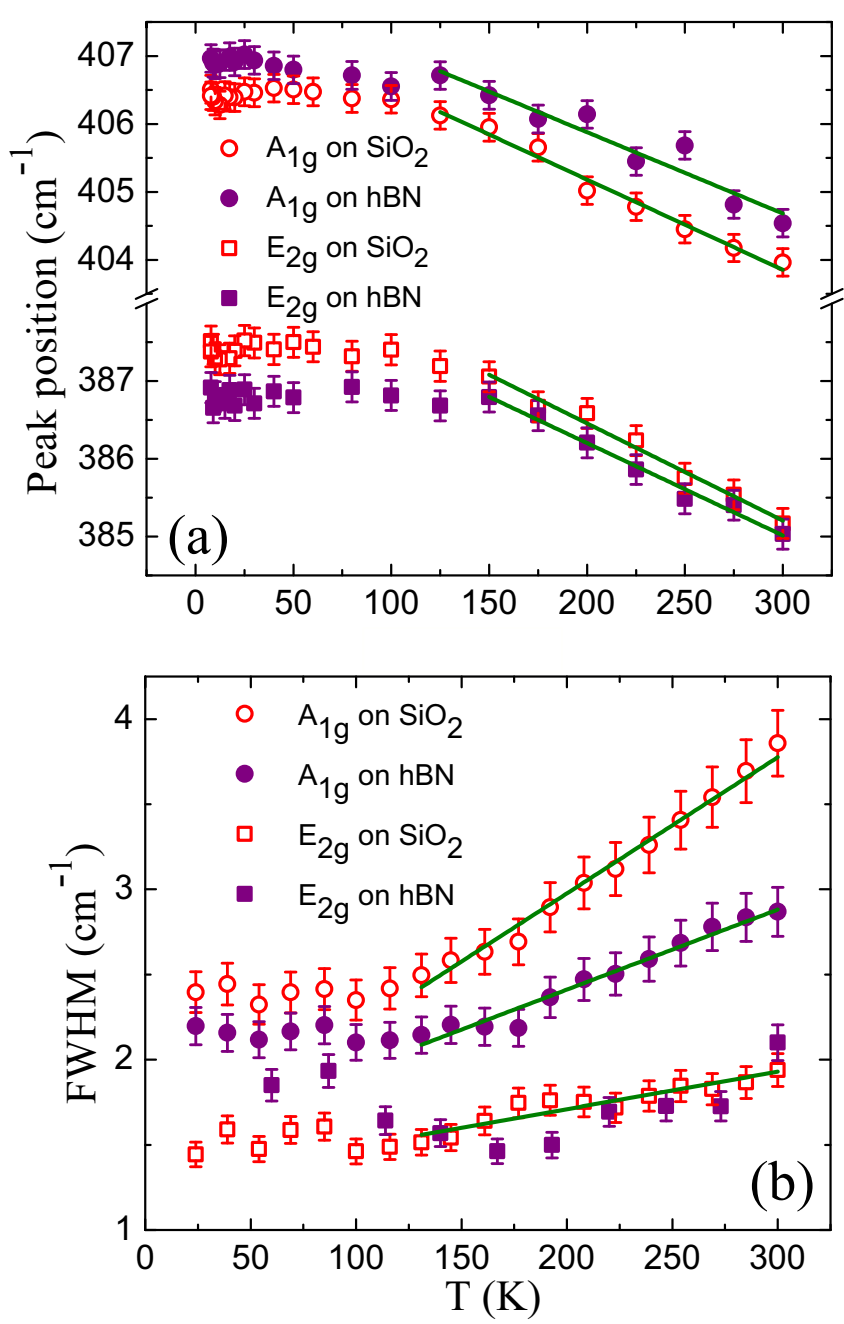

FIG. 5. Plots of (a) the peak positions and (b) the FWHM of the $A_{1 g}$ and $E_{2 g}^{1}$ Raman modes of $\mathrm{MoS}_{2}$ on $\mathrm{hBN}$ and $\mathrm{SiO}_{2}$ substrates-the data were taken on sample2. The green solid lines are the linear fits to experimental data for $T>100 \mathrm{~K}$.

$\mathrm{MoS}_{2}$ look very similar to those of SL MoS 2 [Figs. 4(c) and 4(d)], in agreement with our experiment.

\section{Effect of substrate on doping and strain levels in $\mathrm{MoS}_{\mathbf{2}}$}

The data discussed until now were obtained on $\mathrm{MoS}_{2}$ on a $\mathrm{SiO}_{2}$ substrate. We now turn to probe the effect of substrate on the Raman modes. We have identified the single layer $\mathrm{MoS}_{2}$ by comparing Raman spectra of $\mathrm{SL} \mathrm{MoS}_{2}$ on a $\mathrm{SiO}_{2}$ with $\mathrm{MoS}_{2}$ on hBN in sample2 showed in the Appendix section Fig. 12. We performed temperature dependent Raman spectroscopy measurements in $\mathrm{MoS}_{2}$ on hBN substrate-the data are shown in Fig. 5(a). (We have shown the Raman spectra for sample 2 measured at different temperature between $8 \mathrm{~K}$ to $300 \mathrm{~K}$ in Appendix section Fig. 13.) The data show that the $E_{2 g}^{1}$ peak gets redshifted for $\mathrm{MoS}_{2}$ on the hBN substrate as compared to the $\mathrm{SiO}_{2}$ substrate. On the contrary the $A_{1 g}$ peak gets blueshifted for $\mathrm{MoS}_{2}$ on the hBN substrate compared to the case with the $\mathrm{SiO}_{2}$ substrate. We find a linear variation of $E_{2 g}^{1}$ and $A_{1 g}$ peak frequencies with temperature above $100 \mathrm{~K}$ on the hBN substrate while at low temperatures, the peak 
frequencies and FWHM of both the $E_{2 g}^{1}$ and $A_{1 g}$ saturate. The fact that both the samples on $\mathrm{SiO}_{2}$ and on $\mathrm{hBN}$ show a saturation of Raman shift and FWHM at similar $T$ scale points to an intrinsic origin. Finally we observe from Fig. 5(b) that while the FWHM of the $E_{2 g}^{1}$ mode is comparable for sample1 and sample2, the FWHM of the $A_{1 g}$ mode is significantly lower on the hBN substrate than on the $\mathrm{SiO}_{2}$ substrate. In the following section we present our understanding of the origin of these observations.

The induced carrier density and strain in $\mathrm{MoS}_{2}$ is substrate dependent. From our electrical transport measurements, we found that the mobility of $\mathrm{SL} \mathrm{MoS}_{2}$ on a $\mathrm{SiO}_{2}$ substrate (on samples prepared in a way similar to sample1) is $\sim 2 \mathrm{~cm}^{2} \mathrm{~V}^{-1} \mathrm{~s}^{-1}$ at $100 \mathrm{~K}$ while that of $\mathrm{SL} \mathrm{MoS}_{2}$ on hBN (obtained on samples similar to sample2) is $\sim 20 \mathrm{~cm}^{2} \mathrm{~V}^{-1} \mathrm{~s}^{-1}$ [61]. The impurity number densities extracted from conductance fluctuation spectroscopy measurements for these two classes of samples are $3.5 \times 10^{12} \mathrm{~cm}^{-2} \mathrm{eV}^{-1}$ and $1.8 \times$ $10^{10} \mathrm{~cm}^{-2} \mathrm{eV}^{-1}$, respectively [61].

The higher impurity concentration of $\mathrm{SL} \mathrm{MoS}_{2}$ on a $\mathrm{SiO}_{2}$ substrate effectively corresponds to a large electron doping. Consistently, the FWHM of the $A_{1 g}$ peak is larger on the $\mathrm{SiO}_{2}$ substrate than on the hBN substrate [Fig. 5(b)]. In order to compute the effects of the electron doping on the Raman modes, we explicitly add a fraction of electron in the unit cell of SL MoS 2 in our calculations. We find that the $A_{1 g}$ mode softens significantly (by $\sim-0.4 \mathrm{~cm}^{-1}$ for $0.003 \mathrm{e} /$ cell) with electron doping in agreement with large electron-phonon coupling strength corresponding to this mode [62]. In sharp contrast, the $E_{2 g}^{1}$ mode is practically independent of doping (hardens by $\sim 0.08 \mathrm{~cm}^{-1}$ for $0.003 \mathrm{e} /$ cell). The temperature coefficient of the FWHM of the Raman modes of hBN-MoS $\mathrm{S}_{2}$ sample actually compares well with the theoretically calculated slopes from the three-phonon process. On the contrary, on a $\mathrm{SiO}_{2}$ substrate, the experimental values are not well reproduced by our calculations, which yield significantly smaller estimates. In the case of hBN substrate charge impurity concentration is two orders of magnitude lower so electron-phonon processes are less pronounced. The threephonon process becomes the primary lifetime determining mechanism in the case of $\mathrm{hBN}$-substrate device.

On the other hand, the strain induced due to $\mathrm{hBN}$ on $\mathrm{MoS}_{2}$ is large as compared to that by $\mathrm{SiO}_{2}$. The lattice constant difference is more than 20 percent between $\mathrm{hBN}$ and $\mathrm{MoS}_{2}$. As the heterostructures are made by van der Waals interaction, we do not expect the strain is generated by lattice mismatch. Rather we believe the strain arises from deformed heterostructure due to fabrication. In our case we have prepared the heterostructure by stacking $\mathrm{MoS}_{2}$ on pretransferred hBN flake by dry PDMS transfer technique. In this process the air has been trapped in the interface of the heterostructure. As we anneal our sample through heating in vacuum at about $300^{\circ} \mathrm{C}$, the $\mathrm{MoS}_{2}$ gets stretched by cleaning up most of the interface area and deformed the hBN. After annealing the $\mathrm{MoS}_{2}$ tries to relax but it gets held by the deformed hBN. This does not allow the $\mathrm{MoS}_{2}$ to relax completely. As a result some residual strain sustain on the $\mathrm{MoS}_{2}$. Xu Han et al. show that in SL $\mathrm{MoS}_{2}$ the strain can be up to $0.6 \%$ in the presence of hBN environment [63]. To investigate the effects of uniaxial compressive strain on the high-frequency Raman modes, we apply
$0.1 \%$ to $0.4 \%$ strain to the unit cell of $\mathrm{SL} \mathrm{MoS}_{2}$ and compute the phonon mode frequencies using DFPT. We find that for $\mathrm{MoS}_{2}$ on hBN substrate, the $E_{2 g}^{1}$ mode softens significantly $\left(\sim-1.3 \mathrm{~cm}^{-1}\right)$ more than the $A_{1 g}\left(\sim-0.4 \mathrm{~cm}^{-1}\right) . \mathrm{SiO}_{2}$ as a substrate induces less strain, which results in a stiffer $E_{2 g}^{1}$ mode compared to that of hBN. The combined effect of these two phenomenon-namely higher electron doping levels on $\mathrm{SiO}_{2}$ substrate and higher strain on hBN substrate-provide a natural explanation of the experimentally observed redshift for the $E_{2 g}^{1}$ peak and blueshift in the $A_{1 g}$ peak for $\mathrm{MoS}_{2}$ on the hBN substrate as compared to that on the $\mathrm{SiO}_{2}$ substrate [see Fig. 5(a)]. Interestingly, irrespective of the substrate, the temperature dependent Raman shift shows similar slopes overt the temperature range $100 \mathrm{~K}$ to $300 \mathrm{~K}$ signifying that the doping and strain does not play an important role in determining the temperature dependence of Raman shift (Table I). This strongly supports our explanation of this trend using only intrinsic anharmonic processes.

\section{CONCLUSION}

We have performed a detailed study of temperature on Raman active modes in $\mathrm{MoS}_{2}$ and analyzed the effects of strain and electronic doping imposed by the substrate. Both the Raman shift and FWHM shows linear temperature dependence $T>100 \mathrm{~K}$; below $T<100 \mathrm{~K}$ they became independent of the temperature. Using first principle based calculations, we show that the observed temperature dependence of the Raman shift on SL and on NBL $\mathrm{MoS}_{2}$ arises from both three-phonon and four-phonon processes while the lifetime of these phonon modes primarily arises from the three-phonon process for the in-plane mode while for the outof plane mode electron-phonon process plays an important role too. The higher order phonon processes are present in the system but the lifetime of those higher-order processes is much longer than the three-phonon process due to much lower scattering probabilities and momentum and energy conservation rules. The higher value of FWHM in the out-of-plane vibrational mode as compared to that of the in-plane vibration is consistent with a scenario with a three-phonon process. To understand the contribution of other extrinsic effects like the presence of impurities and strain due to substrate on Raman shifts and Raman modes, a comparison of the data on samples fabricated on a $\mathrm{hBN}$ and on a $\mathrm{SiO}_{2}$ substrate has been performed. The theoretical calculations suggest that the observed differences arise from a larger strain and lower density of impurities on a hBN substrate. Lastly irrespective of sample quality, strain of the temperature coefficient of Raman shift for in-plane and out-of-plane component is constant and it is linear throughout the temperature range $300 \mathrm{~K}$ to about $125 \mathrm{~K}$. Below $100 \mathrm{~K}$ the Raman shift became independent of temperature and saturates. This extrinsic effect plays a static role in Raman shift throughout the temperature range.

\section{ACKNOWLEDGMENTS}

A.B. acknowledges financial support from SERB, DST, Govt. of India and Indo-French Centre for the Promotion 
of Advanced Research (CEFIPRA) Grant No. 5304-3 and supports under FIST program, DST. The authors thank Supercomputer Education and Research Centre at IISc for providing computational resources.

\section{APPENDIX}

\section{Full range Raman spectra and identification of layer number on $\mathrm{SiO}_{2}$ substrate}

In our main text, we have concentrated on the in-plane $E_{2 g}$ and out-of-plane $A_{1 g}$ vibrational modes. We have analyzed the Raman spectra only in the range of $370 \mathrm{~cm}^{-1}$ to $420 \mathrm{~cm}^{-1}$. But our measurements consist of the spectra ranges $200 \mathrm{~cm}^{-1}$ to $800 \mathrm{~cm}^{-1}$ for all the temperatures. In Fig. 6 we have presented a full range spectra for $\mathrm{SL} \mathrm{MoS}_{2}$ on $\mathrm{SiO}_{2}$ substrate at $300 \mathrm{~K}$.

We have identified a single layer (SL) and natural bilayer (NBL) $\mathrm{MoS}_{2}$ sample through Raman and PL spectra. In Fig. 7(a) Raman spectra have been shown for SL and NBL samples. Raman spectra is fitted with two Lorentzian to identify the peak position and to calculate the difference in Raman shift between two peaks. We found that the difference in Raman shifts are 19.5 and $22 \mathrm{~cm}^{-1}$, respectively, shown in Fig. 7(b) which are comparable to the previous report from the literature [14]. On the other hand, the comparative PL spectra identified the SL and NBL samples. SL has a much higher PL intensity than the NBL one shown in Fig. 7(c).

We also have identified SL and NBL with low-frequency Raman measurement. In the case of the SL sample, interlayer shear mode (ISM) and layer breathing mode (LBM) are absent while on the bilayer sample ISM and LBM are present at about $20 \mathrm{~cm}^{-1}$ and $40 \mathrm{~cm}^{-1}$, respectively. The presence of interlayer modes confirms the layer number of the sample shown in Fig. 8.

\section{Results obtained from Voigt fitting to the spectrum}

We have fitted our experimental result with Lorentzian in our paper. We have also used Voigt fitting to determine the peak position and FWHM. Peak position with temperature

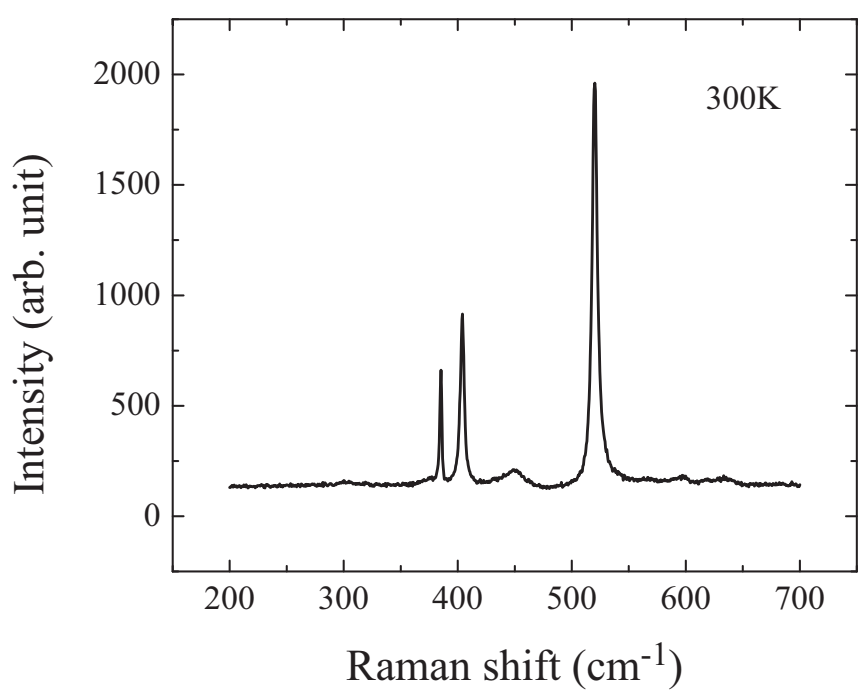

FIG. 6. Raman spectrum for $\mathrm{SL} \mathrm{MoS}_{2}$ on $\mathrm{SiO}_{2}$ substrate at $300 \mathrm{~K}$.
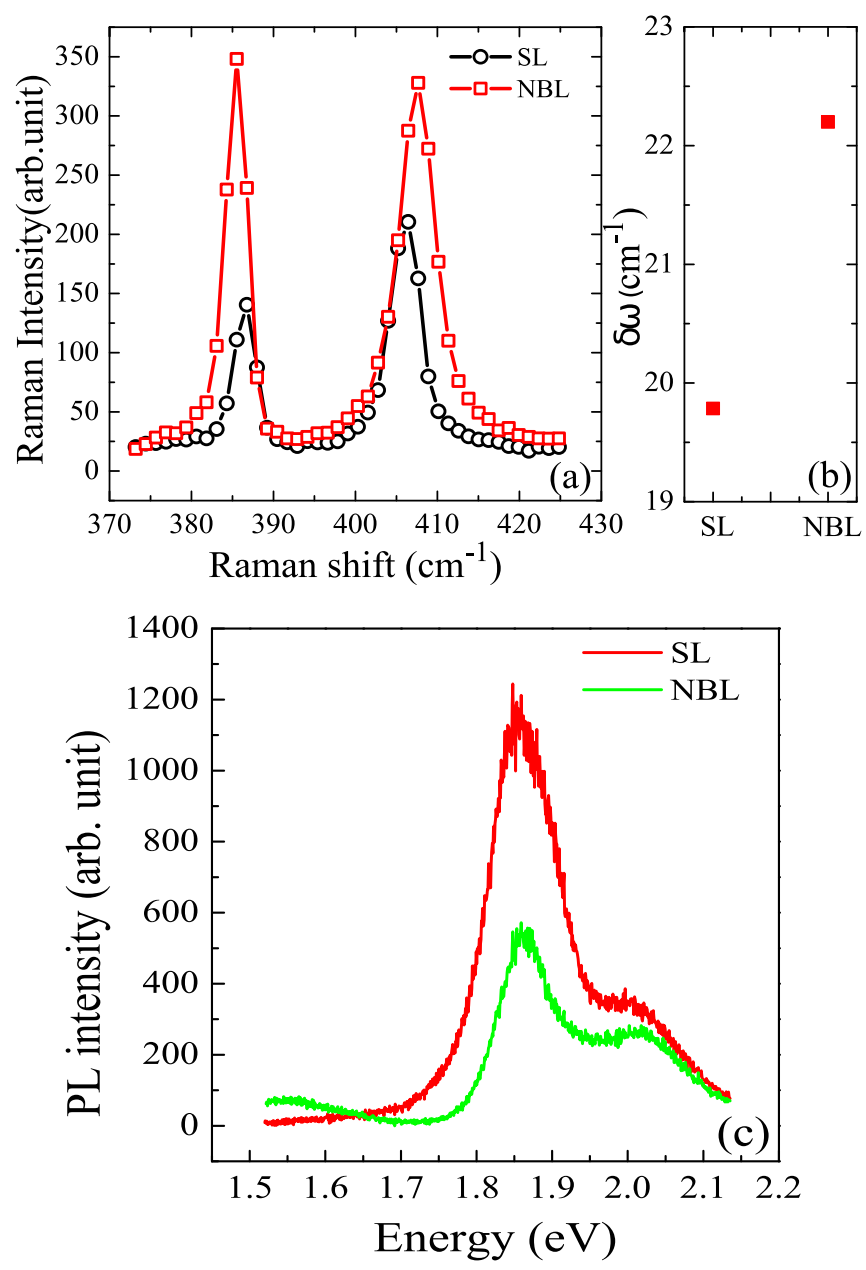

FIG. 7. (a) Raman spectrum for SL and NBL $\mathrm{MoS}_{2}$ on $\mathrm{SiO}_{2}$ substrate at room temperature. (b) Raman shift $\delta \omega$ measured at SL and $\mathrm{NBL} \mathrm{MoS}_{2}$ on $\mathrm{SiO}_{2}$ substrate. (c) Photoluminescence spectra for $\mathrm{SL}$ and $\mathrm{NBL} \mathrm{MoS}_{2}$ on $\mathrm{SiO}_{2}$ substrate at room temperature.

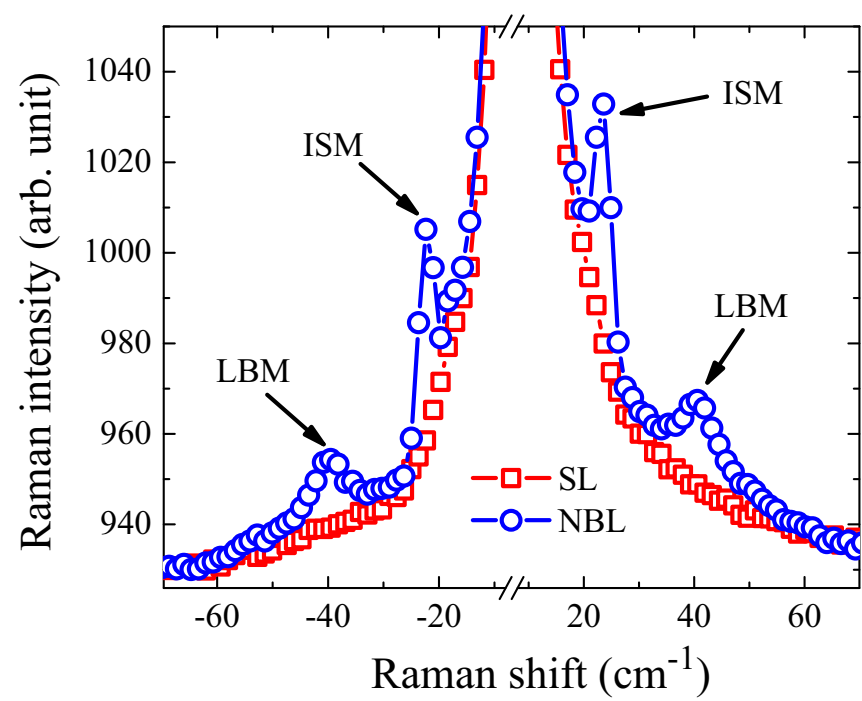

FIG. 8. Raman spectra at room temperature in the low frequency region for $\mathrm{SL}$ and $\mathrm{NBL} \mathrm{MoS}_{2}$ on $\mathrm{SiO}_{2}$. We have marked the peak position of interlayer shear mode and layer breathing mode. 

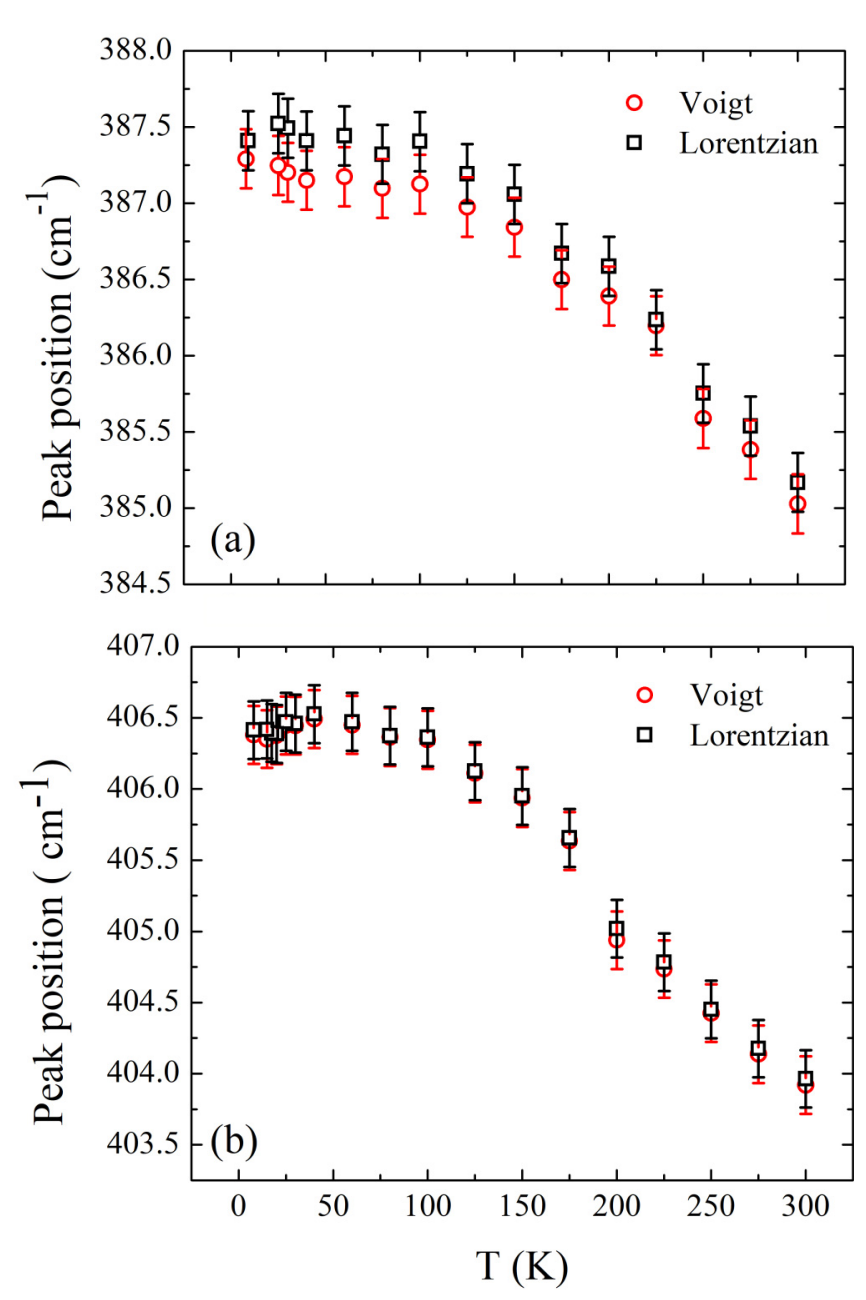

FIG. 9. Plot of peak position with temperature for SL $\mathrm{MoS}_{2}$ on $\mathrm{SiO}_{2}$ by using Voigt and Lorentzian fitting to the Raman spectra for (a) $E_{2 g}$, (b) $A_{1 g}$ mode.

obtained from Voigt and Lorentzian fits are very similar for both $E_{2 g}$ and $A_{1 g}$ peaks shown in Figs. 9(a) and 9(b), respectively. We found that in the case of Voigt fitting both Lorentzian width and Gaussian width contribute to the Voigt width and as a result, we end up with a minimum FWHM for $E_{2 g}$ and $A_{1 g}$ peak which is about $1.8 \mathrm{~cm}^{-1}$ and $2.5 \mathrm{~cm}^{-1}$, respectively, while from Lorentzian fit to our Raman spectra the minimum FWHM is about $1.45 \mathrm{~cm}^{-1}$ and $2.3 \mathrm{~cm}^{-1}$, respectively, shown in Figs. 10(a) and 10(b), respectively. In both Lorentzian and Voigt fitting FWHM follows the same trends with the temperature.

We have plotted the intensity of the Raman peaks for $E_{2 g}$ and $A_{1 g}$ mode for SL $\mathrm{MoS}_{2}$ on $\mathrm{SiO}_{2}$ substrate with temperature in Fig. 11. The peak intensity for $E_{2 g}$ peak remains almost unchanged with temperature while $A_{1 g}$ peak intensity decreases as we increase the temperature.

\section{Detection of SL MoS $\mathrm{Mn}_{2}$ hBN substrate and temperature-dependent Raman spectra}

We have identified SL $\mathrm{MoS}_{2}$ on the hBN substrate and compared the Raman spectra with the $\mathrm{SiO}_{2}$ substrate sample shown in Fig. 12(a) and calculated the peak position dif-
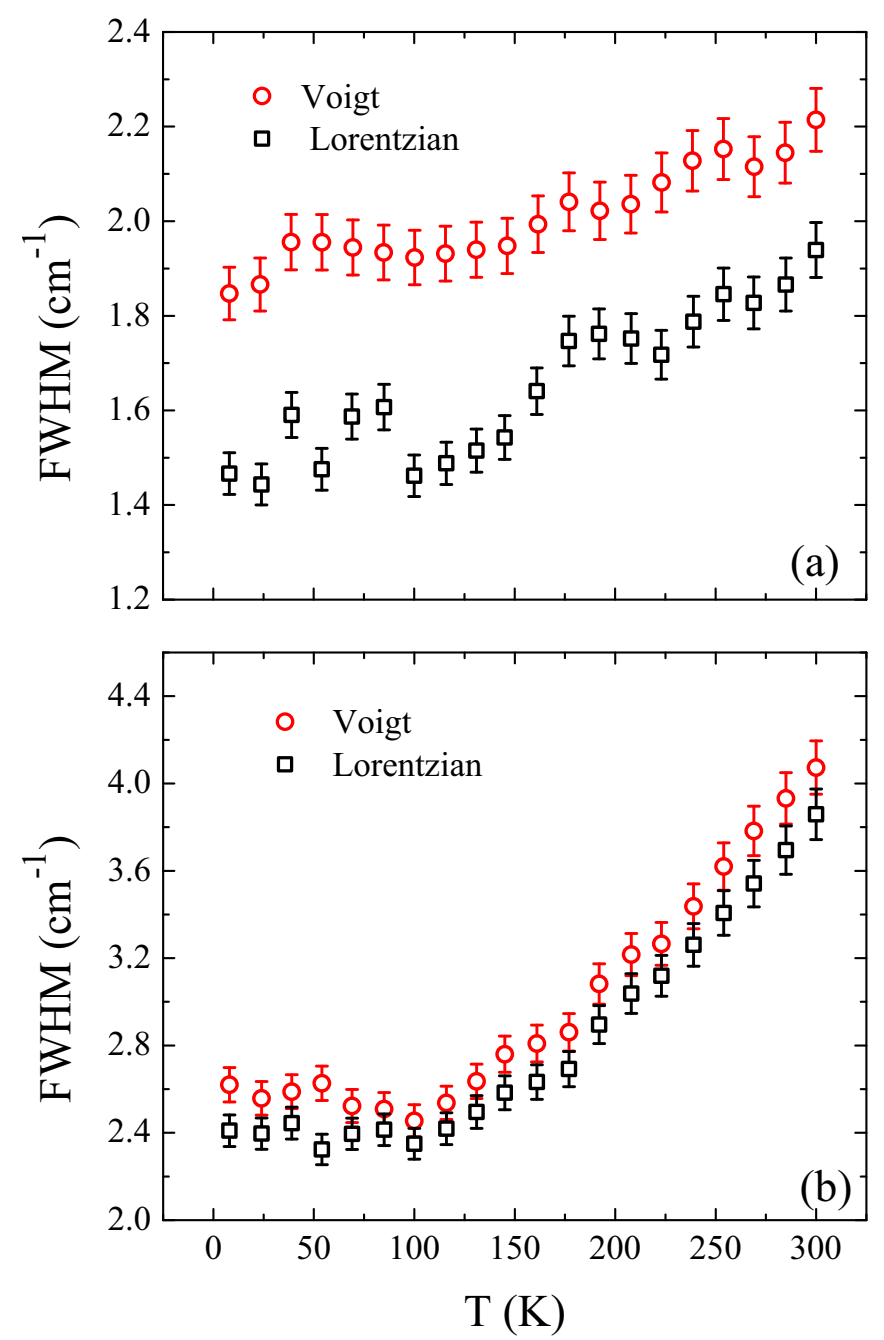

FIG. 10. Plot of FWHM with temperature for $\mathrm{SL} \mathrm{MoS}_{2}$ on $\mathrm{SiO}_{2}$ by using Voigt and Lorentzian fitting to the Raman spectra for (a) $E_{2 g}$, (b) $A_{1 g}$ mode.

ference. We found that the peak position difference is 18.8 $\mathrm{cm}^{-1}$ and $19.5 \mathrm{~cm}^{-1}$ for $\mathrm{SiO}_{2}$ and hBN substrate sample, respectively, shown in Fig. 12(b).

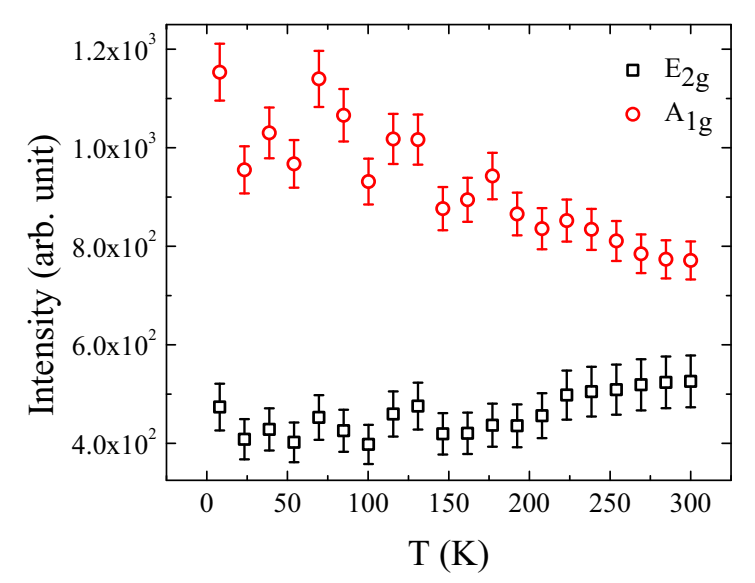

FIG. 11. Plot of peak intensity with temperature for $E_{2 g}$ and $A_{1 g}$ mode in $\mathrm{SL} \mathrm{MoS}_{2}$ on $\mathrm{SiO}_{2}$ substrate. 

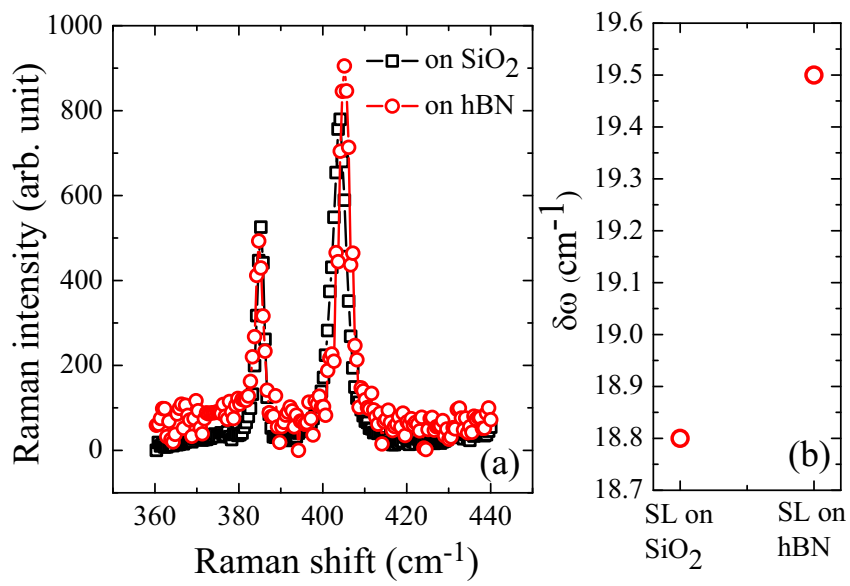

FIG. 12. Plot of (a) High frequency Raman spectrum of SL MoS on $\mathrm{SiO}_{2}$ and hBN substrate. (b) High frequency Raman shift $\delta \omega$ measured at $\mathrm{SL} \mathrm{MoS} 2$ on $\mathrm{SiO}_{2}$ and hBN substrate.

We have presented the temperature dependent Raman spectra for hBN substrate SL $\mathrm{MoS}_{2}$ on sample2 from $300 \mathrm{~K}$ to $8 \mathrm{~K}$ temperature range shown in Fig. 13. The spectra shows the blueshift with temperature until $100 \mathrm{~K}$ and then saturates below that temperature. The FWHM decreases

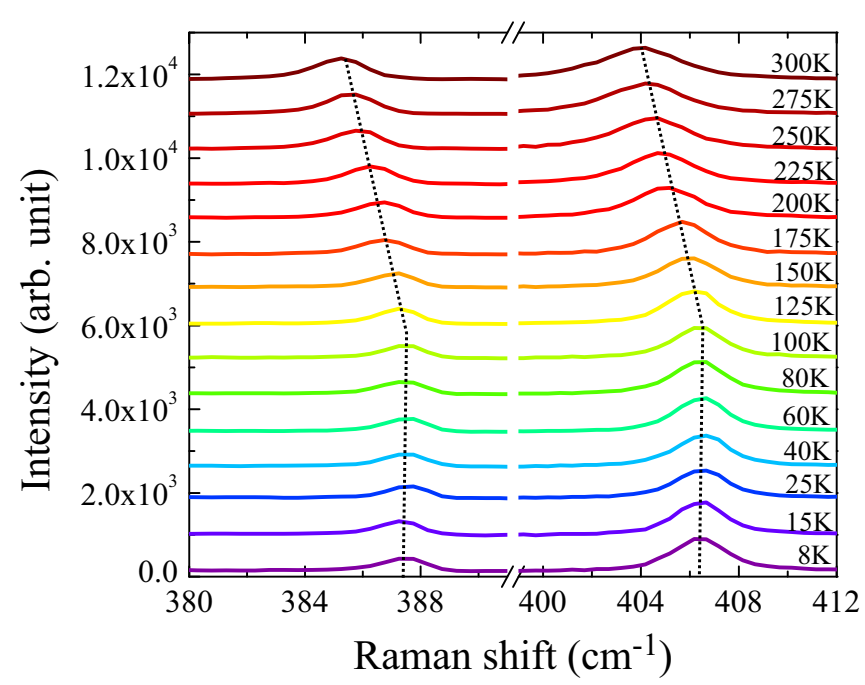

FIG. 13. Raman spectra of hBN substrate $\mathrm{SL} \mathrm{MoS}_{2}$ on sample2 measured at different temperatures between $8 \mathrm{~K}$ and $300 \mathrm{~K}$. The dotted lines are the guide for the eye for marking the evolution of Raman shift with temperature.

with decreasing temperature can also be noticed from the data.
[1] K. F. Mak, C. Lee, J. Hone, J. Shan, and T. F. Heinz, Phys. Rev. Lett. 105, 136805 (2010).

[2] B. Radisavljevic, A. Radenovic, J. Brivio, V. Giacometti, and A. Kis, Nat. Nanotechnol. 6, 147 (2011).

[3] K. F. Mak, K. He, C. Lee, G. H. Lee, J. Hone, T. F. Heinz, and J. Shan, Nat. Mater. 12, 207 (2013).

[4] W. Bao, X. Cai, D. Kim, K. Sridhara, and M. S. Fuhrer, Appl. Phys. Lett. 102, 042104 (2013).

[5] Y. Zhang, J. Ye, Y. Matsuhashi, and Y. Iwasa, Nano Lett. 12, 1136 (2012).

[6] J. Lu, O. Zheliuk, I. Leermakers, N. F. Yuan, U. Zeitler, K. T. Law, and J. Ye, Science 350, 1353 (2015).

[7] K. Taniguchi, A. Matsumoto, H. Shimotani, and H. Takagi, Appl. Phys. Lett. 101, 042603 (2012).

[8] X. Liu, T. Galfsky, Z. Sun, F. Xia, E.-c. Lin, Y.-H. Lee, S. KenaCohen, and V. Menon, Nat. Photonics 9, 30 (2014).

[9] K. F. Mak, K. He, J. Shan, and T. F. Heinz, Nat. Nanotechnol. 7, 494 (2012).

[10] X. Xu, W. Yao, D. Xiao, and T. F. Heinz, Nat. Phys. 10, 343 (2014).

[11] J. Hong, Z. Hu, M. Probert, K. Li, D. Lv, X. Yang, L. Gu, N. Mao, Q. Feng, L. Xie et al., Nat. Commun. 6, 6293 (2015).

[12] S. Tongay, J. Suh, C. Ataca, W. Fan, A. Luce, J. S. Kang, J. Liu, C. Ko, R. Raghunathanan, J. Zhou et al., Sci. Rep. 3, 2657 (2013).

[13] Z.-Y. Ong and M. V. Fischetti, Phys. Rev. B 88, 165316 (2013).

[14] S. Dubey, S. Lisi, G. Nayak, F. Herziger, V.-D. Nguyen, T. Le Quang, V. Cherkez, C. Gonzalez, Y. J. Dappe, K. Watanabe et al., ACS Nano 11, 11206 (2017).

[15] A. Molina-Sánchez, D. Sangalli, K. Hummer, A. Marini, and L. Wirtz, Phys. Rev. B 88, 045412 (2013).
[16] D. W. Latzke, W. Zhang, A. Suslu, T.-R. Chang, H. Lin, H.-T. Jeng, S. Tongay, J. Wu, A. Bansil, and A. Lanzara, Phys. Rev. B 91, 235202 (2015).

[17] G. Wang, A. Chernikov, M. M. Glazov, T. F. Heinz, X. Marie, T. Amand, and B. Urbaszek, Rev. Mod. Phys. 90, 021001 (2018).

[18] K. Golasa, M. Grzeszczyk, P. Leszczynski, C. Faugeras, A. Nicolet, A. Wysmolek, M. Potemski, and A. Babinski, Appl. Phys. Lett. 104, 092106 (2014).

[19] Y. Lin, X. Ling, L. Yu, S. Huang, A. L. Hsu, Y.-H. Lee, J. Kong, M. S. Dresselhaus, and T. Palacios, Nano Lett. 14, 5569 (2014).

[20] R. Yan, S. Bertolazzi, J. Brivio, T. Fang, A. Konar, A. G. Birdwell, N. Nguyen, A. Kis, D. Jena, and H. G. Xing, arXiv:1211.4136.

[21] C. Rice, R. Young, R. Zan, U. Bangert, D. Wolverson, T. Georgiou, R. Jalil, and K. Novoselov, Phys. Rev. B 87, 081307 (2013).

[22] Y. Wang, C. Cong, C. Qiu, and T. Yu, Small 9, 2857 (2013).

[23] A. Castellanos-Gomez, R. Roldán, E. Cappelluti, M. Buscema, F. Guinea, H. S. van der Zant, and G. A. Steele, Nano Lett. 13, 5361 (2013).

[24] M. Boukhicha, M. Calandra, M.-A. Measson, O. Lancry, and A. Shukla, Phys. Rev. B 87, 195316 (2013).

[25] M. Yang, X. Cheng, Y. Li, Y. Ren, M. Liu, and Z. Qi, Appl. Phys. Lett. 110, 093108 (2017).

[26] S. Sahoo, A. P. Gaur, M. Ahmadi, M. J.-F. Guinel, and R. S. Katiyar, J. Phys. Chem. C 117, 9042 (2013).

[27] L. F. Huang, P. L. Gong, and Z. Zeng, Phys. Rev. B 90, 045409 (2014).

[28] D. J. Late, S. N. Shirodkar, U. V. Waghmare, V. P. Dravid, and C. Rao, ChemPhysChem 15, 1592 (2014). 
[29] X. Liu, G. Zhang, Q.-X. Pei, and Y.-W. Zhang, Appl. Phys. Lett. 103, 133113 (2013).

[30] W. Li, J. Carrete, and N. Mingo, Appl. Phys. Lett. 103, 253103 (2013).

[31] M. Thripuranthaka, R. V. Kashid, C. Sekhar Rout, and D. J. Late, Appl. Phys. Lett. 104, 081911 (2014).

[32] S. Najmaei, P. M. Ajayan, and J. Lou, Nanoscale 5, 9758 (2013).

[33] T. Yang, X. Huang, H. Zhou, G. Wu, and T. Lai, Opt. Express 24, 12281 (2016).

[34] N. A. Lanzillo, A. Glen Birdwell, M. Amani, F. J. Crowne, P. B. Shah, S. Najmaei, Z. Liu, P. M. Ajayan, J. Lou, M. Dubey et al., Appl. Phys. Lett. 103, 093102 (2013).

[35] A. Taube, J. Judek, A. Lapiénska, and M. Zdrojek, ACS Appl. Mater. Interfaces 7, 5061 (2015).

[36] R. Yan, J. R. Simpson, S. Bertolazzi, J. Brivio, M. Watson, X. Wu, A. Kis, T. Luo, A. R. Hight Walker, and H. G. Xing, ACS Nano 8, 986 (2014).

[37] S. Baroni, S. De Gironcoli, A. Dal Corso, and P. Giannozzi, Rev. Mod. Phys. 73, 515 (2001).

[38] A. Castellanos-Gomez, M. Buscema, R. Molenaar, V. Singh, L. Janssen, H. S. J. van der Zant, and G. A. Steele, 2D Materials 1, 011002 (2014).

[39] C. R. Dean, A. F. Young, I. Meric, C. Lee, L. Wang, S. Sorgenfrei, K. Watanabe, T. Taniguchi, P. Kim, K. L. Shepard et al., Nat. Nanotechnol. 5, 722 (2010).

[40] L. Paulatto, F. Mauri, and M. Lazzeri, Phys. Rev. B 87, 214303 (2013).

[41] W. Kohn and L. J. Sham, Phys. Rev. 140, A1133 (1965).

[42] P. Giannozzi, O. Andreussi, T. Brumme, O. Bunau, M. B. Nardelli, M. Calandra, R. Car, C. Cavazzoni, D. Ceresoli, M. Cococcioni et al., J. Phys.: Condens. Matter 29, 465901 (2017).

[43] P. Giannozzi, S. Baroni, N. Bonini, M. Calandra, R. Car, C. Cavazzoni, D. Ceresoli, G. L. Chiarotti, M. Cococcioni, I. Dabo et al., J. Phys.: Condens. Matter 21, 395502 (2009).

[44] D. Hamann, Phys. Rev. B 88, 085117 (2013).
[45] M. Schlipf and F. Gygi, Comput. Phys. Commun. 196, 36 (2015).

[46] J. P. Perdew, K. Burke, and M. Ernzerhof, Phys. Rev. Lett. 77, 3865 (1996).

[47] A. Molina-Sánchez and L. Wirtz, Phys. Rev. B 84, 155413 (2011).

[48] H. Li, Q. Zhang, C. C. R. Yap, B. K. Tay, T. H. T. Edwin, A. Olivier, and D. Baillargeat, Adv. Funct. Mater. 22, 1385 (2012).

[49] R. Saito, Y. Tatsumi, S. Huang, X. Ling, and M. Dresselhaus, J. Phys.: Condens. Matter 28, 353002 (2016).

[50] C. Lee, H. Yan, L. E. Brus, T. F. Heinz, J. Hone, and S. Ryu, ACS Nano 4, 2695 (2010).

[51] L. Su, Y. Zhang, Y. Yu, and L. Cao, Nanoscale 6, 4920 (2014).

[52] N. V. Surovtsev and I. N. Kupriyanov, Crystals 7, 239 (2017).

[53] M. Balkanski, R. Wallis, and E. Haro, Phys. Rev. B 28, 1928 (1983).

[54] J. Menéndez and M. Cardona, Phys. Rev. B 29, 2051 (1984).

[55] P. Klemens, Phys. Rev. 148, 845 (1966).

[56] N. Wakabayashi, H. Smith, and R. Nicklow, Phys. Rev. B 12, 659 (1975).

[57] T. Feng, L. Lindsay, and X. Ruan, Phys. Rev. B 96, 161201 (2017).

[58] W. M. Parkin, A. Balan, L. Liang, P. M. Das, M. Lamparski, C. H. Naylor, J. A. Rodríguez-Manzo, A. C. Johnson, V. Meunier, and M. Drndiéc, ACS Nano 10, 4134 (2016).

[59] M. Buscema, G. A. Steele, H. S. van der Zant, and A. Castellanos-Gomez, Nano Res. 7, 561 (2014).

[60] S. Mignuzzi, A. J. Pollard, N. Bonini, B. Brennan, I. S. Gilmore, M. A. Pimenta, D. Richards, and D. Roy, Phys. Rev. B 91, 195411 (2015).

[61] S. Sarkar, A. Bid, K. L. Ganapathi, and S. Mohan, Phys. Rev. B 99, 245419 (2019).

[62] B. Chakraborty, A. Bera, D. Muthu, S. Bhowmick, U. V. Waghmare, and A. Sood, Phys. Rev. B 85, 161403 (2012).

[63] X. Han, J. Lin, J. Liu, N. Wang, and D. Pan, J. Phys. Chem. C 123, 14797 (2019). 